\title{
A New Technique of Auction Based Spectrum Allocation for CR Network
}

\author{
Jyoti Shivshankar Swami ${ }^{1}$, Sangita Nikumbh ${ }^{2}$ \\ ${ }^{1}$ Department of Electronics \& Telecommunication Engineering, Yadavrao Tasgaonkar College of Engg. \& Technology, Bhivpuri, Karjat \\ ${ }^{2}$ Professor, Department of Electronics \& Telecommunication Engineering, Yadavrao Tasgaonkar College of Engg. \& Technology, Bhivpuri, \\ Karjat
}

\begin{abstract}
The survey of Federal Communications Commission (FCC) in 2002, shows that spectrum access is more significant problem than physical scarcity of spectrum. With many technological advances in the field of wireless communication the demand for radio spectrum has tremendously increased and with the standardization of MBMS (multimedia broadcast and multicast services) it has gained significant interest in the market. With the diversification of wireless communication services and the proliferation of different wireless network technologies, the demand for radio spectrum is increasing dramatically. However, spectrum measurements indicate that the allocated spectrum is not fully utilized, that is, there exists unused spectrum. Cognitive radio has attracted an increasing amount of interest over the past few years as an effective method of alleviating the spectrum scarcity problem in wireless communications. One of the most promising approaches in cognitive radio is the opportunistic spectrum access, which enables unlicensed users to access licensed frequency bands that are detected to be idle. We develop a truthful and efficient combinatorial auction scheme under a novel spectrum allocation model. Once the spectrum is sensed next important step for cognitive radio is allocation of available spectrum (The available spectrum holes, i.e., frequency bands assigned to a primary user but that are vacant in a given place at a given time, can be estimated with spectrum sensing).There are different spectrum allocation mechanism are available such as auction mechanism, first come first serve etc. We use auction mechanism in our project. According to the usage of secondary user in term of time and frequency and available spectrum primary operator $(\mathrm{PO})$ allocate this spectrum on the basis of one time bidding of secondary users. The algorithm approach will be developed using Matlab environment.
\end{abstract}

Keywords: Cognitive radio, combinatorial auction, proliferation, opportunistic spectrum access

\section{Introduction}

The radio frequency spectrum is a natural resource and its efficient use is important issue. The spectrum bands are usually licensed to certain services, such as mobile, TV broadcast and satellite to avoid harmful interference between different networks to affect users. Most spectrum bands are allocated to certain services but worldwide spectrum occupancy measurements show that only some portion of the spectrum bands are used. In the development of future wireless systems, the spectrum utilization functionalities will play a key role due to the scarcity of unoccupied spectrum. Cognitive radios, with the capabilities to sense the operating environment, learn and adapt in real time according to environment creating a form of mesh network, are seen as a promising technology.

Now-a-day, the growing demand of wireless applications has put a lot of constraints on the usage of available radio spectrum which is limited and precious resource. However, a fixed spectrum assignment has lead to under utilization of spectrum as a great portion of licensed spectrum is not effectively utilized. Cognitive Radio (CR) is a promising technology which provides a novel way to improve utilization efficiency of available electromagnetic spectrum. It is able to sense the spectral environment over a wide frequency band and exploit this information to opportunistically provide wireless links that best meet the user communications requirements. Spectrum sensing helps to detect the spectrum holes (unutilized bands of the spectrum) providing high spectral resolution capability. The main objective is to detect spectrum and allocate this available spectrum according to time and frequency usages of secondary users. It sets the priority of secondary users using auction mechanism. In this synopsis, implementation of two dimension spectrum allocation techniques are discussed in detail giving study of spectrum sensing and allocation for better social welfare and spectrum ratio. It gives good opportunity to use cognitive radio to use our natural resources efficiently.

\subsection{Basic Cognitive Cycle}

A basic Cognitive Cycle comprises of following three basic tasks:

I. Spectrum Sensing

II. Spectrum Analysis

III. Spectrum Decision Making

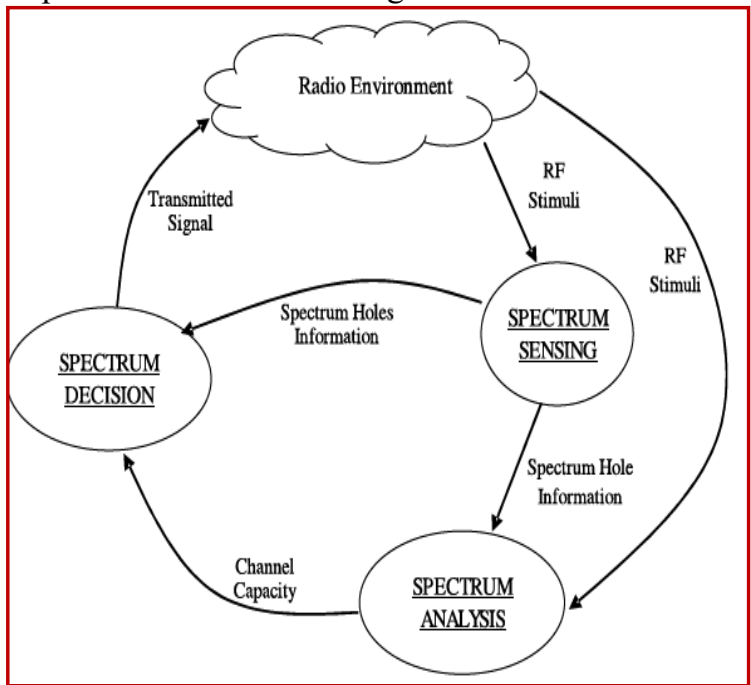

Figure 1: Cognitive Radio cycle 


\section{International Journal of Science and Research (IJSR) \\ ISSN (Online): 2319-7064 \\ Index Copernicus Value (2013): 6.14 | Impact Factor (2014): 5.611}

\section{B. Spectrum Sensing}

It is the capability of Spectrum Sensing to determine, sense and must know about the parameters related to the radio channel, spectrum availability and power that is transmitted, interference and noise and the environment in which radio operation occurs, user necessities and applications, existing networks infrastructures and nodes, confined policies and other operating limitations. Sensing is done across Geographical Space, Frequency, Time, Phase and code

\section{Spectrum Analysis}

The Analysis of spectrum is based on spectrum sensing the external and internal radio environment in which several factors analyzed the situation such as radio frequency spectrum use by adjacent devices, user behavior and network existing conditions and determining the optimal communication protocol and variation of frequency or channel accordingly which is called channel estimation.

\section{Spectrum Decision Making}

Spectrum decision making is related to reconfiguration of the channel and for changing the mobile environments constantly adapting protocols are needed and output power adjustment or even alternative parameters related to the transmission (like different channel coding schemes and Variable symbol rates and also the low to high order of these schemes ) and features of the Cognitive Radio devices and for increasing the capacity or range extension the CR use multiple antennas to minimize interference.

\section{Literature Survey}

In [1] Changle Li elaborated, Two Dimension Spectrum Allocation for Cognitive Radio Network in which he propose to tackle the dynamic spectrum access problem in cognitive radio (CR) networks with time-frequency flexibility requirements. In this paper he only concentrate on the problem how the PO sells the acquired spectrum to SUs so as to maximize the social welfare (the sum of the declared valuation of the granted SUs) with time-frequency variant and heterogeneous requirements.

In [2] A Survey of Spectrum Sensing Algorithms for Cognitive Radio Applications" by Tevfik Y"ucek and H"useyin Arslan in his paper explain different terms like Cognitive radio, spectrum sensing, dynamic spectrum access, multi-Dimensional spectrum sensing, cooperative sensing, radio identification. It elaborate all tech of spectrum sensing In [3] Oh-Soon Shin, Albert M. Chan, H. T. Kung, and Vahid Tarokh explains Design of an OFDM Cooperative Space-Time Diversity System. In this paper explained a space-time cooperative system based on orthogonal frequency division multiplexing (OFDM), which we refer to as a cooperative (CO)-OFDM system.

In [4] "A Strategy-Proof Combinatorial Heterogeneous Channel Auction Framework in Noncooperative Wireless Networks" by Zhenzhe Zheng, Student Member, IEEE, Fan $\mathrm{Wu}$, member, IEEE, and Guihai Chen, Member, IEEE explain practical channel auction mechanism and consider five challenges, including strategy-proofness, channel spatial reusability, channel heterogeneity, bid diversity, and social welfare maximization.

In [5] the Spectrum Auction Framework for Access Allocation in Cognitive Radio Networks is explained. In this paper authors develop an auction-based framework that allows networks to bid for primary and secondary access based on their utilities and traffic demands. The bids are used to solve the access allocation problem.

In [6] the CO-OFDM Transmission System Based on TwoDimensional Permutation is studied. In this paper theory and performance analyses is discussed which increase the capacity and sensitivity of network.

In [7] Dusit Niyato and Ekram Hossain explain Competitive Pricing for Spectrum Sharing in Cognitive Radio Networks: Dynamic Game, Inefficiency of Nash Equilibrium, and Collusion. In which a game-theoretic model is introduced to obtain the optimal pricing for dynamic spectrum sharing in cognitive radio networks.

In [8] Dennis L. Goeckel and Ganesh Ananthaswamy explain orthogonal frequency division modulation system operating over a wireless communication channel effectively.

In [9] Detection performance of cooperative spectrum sensing is studied for data fusion and decision fusion strategies by Saman Atapattu, Chintha Tellambura and Hai Jiang.

In [10] the relay and filter design mechanism is discussed which is useful in orthogonal frequency modulation for cognitive radio network by Donggun Kim, Youngchul Sung and Jihoon Chung.

\section{System Development}




\section{International Journal of Science and Research (IJSR) \\ ISSN (Online): 2319-7064}

Index Copernicus Value (2013): 6.14 | Impact Factor (2014): 5.611

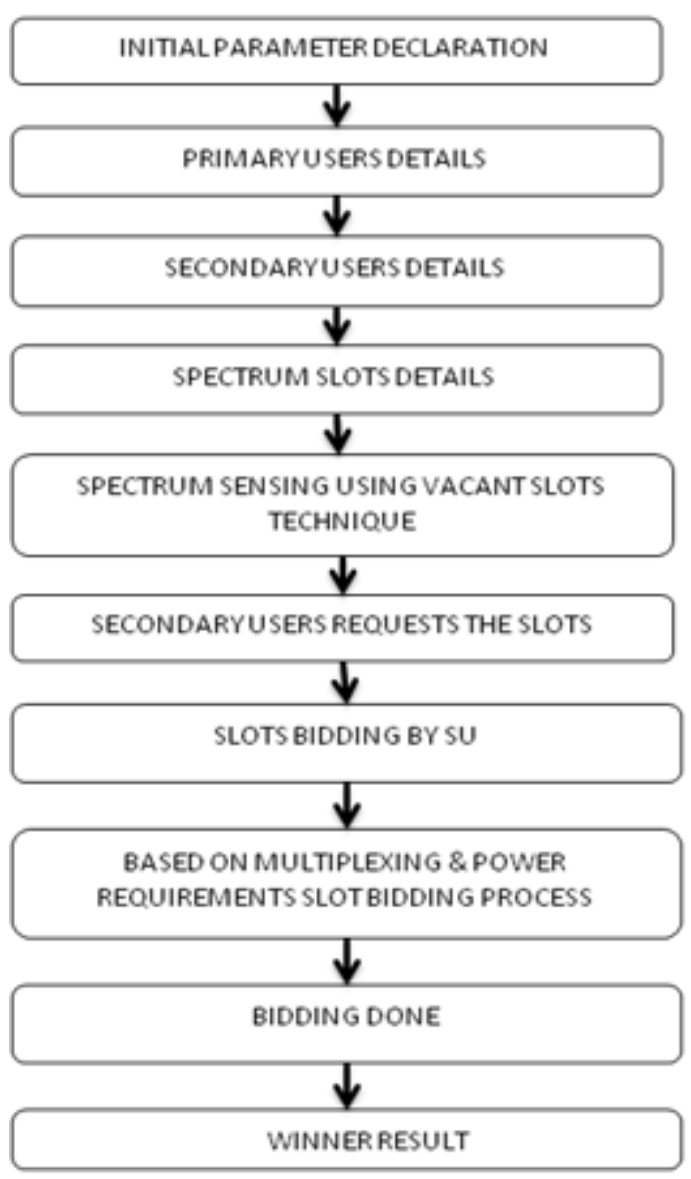

Figure 2: Flow Diagram

The parameters are defined for the channel sensing for PU and SU. The primary user details like opting for the slot or not opting for the slot are mentioned. After analysis of slot details, the available slots for the secondary users are displayed.

The secondary users sense the available slot. Based on the time and having higher power capability (like VCG mechanism), the slots are auctioned to the secondary users. The power spectral density variations are plotted before and after slot allocations. Based on the slot allotment, bidding result is declared as winner or not done.

\section{Performance Evaluation}

This project work is implemented by using matlab $7.10 \mathrm{a}(\mathrm{R}$ 2010 a) to plot various graphs such as power spectral density variations before and after slot allocations and spectrum utilization ratio versus number of secondary users.

\section{Conclusion}

We develop a truthful and efficient combinatorial auction scheme under a novel spectrum allocation model. Based on the time and having higher power capability (like VCG mechanism), the slots are auctioned to the secondary users. Based on the slot allotment, bidding winner is declared. We have developed the proposed system using Matlab environment.

\section{References}

[1] Changle Li,Zhe Liu, Xiaoyan Geng, Mo Dong, Feng Yang, Xiaoying Gan, Xiaohua Tian,and Xinbing Wang,"Two Dimension Spectrum Allocation for Cognitive Radio Networks",IEEE TRANSACTIONS ON WIRELESS COMMUNICATIONS, VOL. 13, NO. 3, MARCH 2014.

[2] T. S. Rappaport, Wireless Communications: Principles and Practice.Prentice-Hall PTR, 2002.

[3] F. S. P. T. Force, "Report of the spectrum efficiency working group,"FCC Spectrum Policy Task Force (SPTF), no. 03-237, Nov. 2002.

[4] L. Sun and W. Wang, "On distribution and limits of information dissemination latency and speed in mobile cognitive radio networks,"in Proc. 2011 IEEE INFOCOM, pp. 246-250.

[5] J. Mitola, "Cognitive radio: an integrated agent architecture for software defined radio," Doctor of Technology, Royal Inst. Technol.(KTH), pp.271-350, 2000.[6] L. Sun and W. Wang, "Understanding the tempo-spatial limits of information dissemination in multi- channel cognitive radio networks," in Proc. 2012 IEEE INFOCOM, pp. 12781286.

[6] L.Sun W. Wang "Understanding the tempo-spatial limits of information in multichannel cognitive radio networks" 2012 IEEE

[7] D. Niyato and E. Hossain, "Competitive pricing for spectrum sharing in cognitive radio networks: dynamic game, inefficiency of Nash equilibrium, and collusion," IEEE J. Sel. Areas Commun., vol. 26, no. 1, pp.192-202, Jan. 2008.

[8] W. Saad, "Coalitional game theory for distributed cooperation in next generation wireless networks," Ph.D. dissertation, University of Oslo,2010.

[9] L. Gao, X. Wang, Y. Xu, and Q. Zhang, "Spectrum trading in cognitive radio networks: a contract-theoretic modeling approach," IEEE J. Sel.Areas Commun., vol. 29, no. 4, pp. 843-855, 2011.

[10] J. Huang, R. Berry, and M. Honig, "Auction-based spectrum sharing,"Mobile Netw. Applications, vol. 11, no. 3, pp. 405-418, 2006

[11] G. Kasbekar and S. Sarkar, "Spectrum auction framework for access allocation in cognitive radio networks," IEEE/ACM Trans. Netw., vol. 18,no. 6, pp. 1841-1854, Dec. 2010.

[12] J. Huang, Z. Han, M. Chiang, and H. Poor, "Auction-based resource allocation for cooperative communications," IEEE J. Sel. Areas Commun.,vol. 26, no. 7, pp. 1226-1237, 2008.

[13] S. Gandhi, C. Buragohain, L. Cao, H. Zheng, and S. Suri, "A general framework for wireless spectrum auctions," in Proc. 2007 IEEE International Symp. New Frontiers Dynamic Spectrum Access Netw., pp. 22-33.

[14] L. Chen, S. Iellamo, M. Coupechoux, and P. Godlewski, "An auction framework for spectrum allocation with interference constraint in cognitive radio networks," in Proc. 2010 IEEE INFOCOM, pp. 1-9. [15] S. Sodagari, A. Attar, and S. Bil'en, "On a truthful mechanism for expiring spectrum sharing in cognitive radio networks," IEEE $J$. Sel.Areas Commun., vol. 29, no. 4, pp. 856-865, 2011.

[15] X. Zhou and H. Zheng, "Trust: a general framework for truthful double spectrum auctions," in Proc. 2009 IEEE INFOCOM, pp. 999-1007. 